\title{
Injectable therapies for knee osteoarthritis
}

\author{
Jack B. Ding ${ }^{1}$, Kevin $\mathrm{Hu}^{2}$ \\ ${ }^{1}$ Faculty of Health and Medical Sciences, Adelaide Medical School, University of Adelaide, Australia \\ ${ }^{2}$ Department of Radiology, Lyell McEwin Hospital, Adelaide, Australia
}

\begin{abstract}
Knee osteoarthritis is a degenerative arthritis that mainly affects older adults. Over time, osteoarthritis can result in significant and sustained discomfort, pain, and disability. Current treatment focuses on the alleviation of pain and functional impairment. While arthroplasty is the definitive management option, it subjects patients to surgical complications, and the possibility of surgical revisions. In addition, many patients are not surgical candidates. Instead, pharmacological therapy is recommended first-line for most patients. On top of pharmacological therapy, there are a range of non-operative procedural options available. However, leading professional guidelines vary in their recommendations for these agents.

Therefore, we present a review of recent randomized controlled trials and meta-analyses on injectable corticosteroids, hyaluronic acid (HA), platelet-rich plasma (PRP), mesenchymal stem cell injections, and ozone therapy. The preliminary data reveal the strongest evidence in favour of corticosteroid injections, although there are promising findings regarding the long-term efficacy of HA and PRP.
\end{abstract}

Key words: osteoarthritis, hyaluronic acid, platelet-rich plasma, intra-articular corticosteroids.

\section{Introduction}

Osteoarthritis (OA) is one of the most frequently diagnosed conditions in medicine, with some estimates suggesting over 27 million US adults suffer from it [1]. While any joint can be inflicted by OA, it more commonly manifests in the lower extremity weight-bearing joints, with an overall lifetime risk of knee OA being around $47 \%$ in females and $40 \%$ in males [1].

Pain is often the chief complaint that individuals with OA present with [2]. The presence of osteoarthritic pain is associated with a reduction in quality of life and is the primary cause of impaired mobility in older adults [3, 4].

Approximately $80 \%$ of people with OA have some level of movement impairment, and around $25 \%$ are severely limited in their activities of daily living [2]. People suffering from OA have primarily attributed their functional impairments to be due to pain, with lesser emphasis on deteriorating ranges of motion or muscle or muscle deconditioning or cognitive factors [5].

Obesity is one of the strongest risk factors for OA, with one calculation suggesting that for every $5 \mathrm{~kg}$ of weight gain, there was a $36 \%$ increased risk of developing knee OA [6]. This dynamic disposes individuals to a vicious cycle, whereby OA pain limits functional mobility, which leads to weight gain, which can aggravate the pain.

The updated International Association for the Study of Pain defines pain as an "an unpleasant sensory and emotional experience associated with, or resembling that associated with, actual or potential tissue damage" [7].

While traditionally regarded as a "non-inflammatory joint disease", OA is now recognized as a low-grade inflammatory disease of the entire joint, affecting the synovium, joint capsule, ligaments, and menisci [8]. These structural changes shift the metabolic pathways of chondrocytes and other cells in joint tissues from a baseline quiescent state to a highly active metabolic state where synovial cells and chondrocytes release and interact with inflammatory cytokines [9].

Over time, this can lead to progressive cartilage damage and formation of osteophytes. Notably, synovial cytokines have been significantly associated with knee pain and poorer function, suggesting that localized in-

\section{Address for correspondence:}

Jack B. Ding, Adelaide Health and Medical Sciences (AHMS), The University of Adelaide, 4 North Terrace, Adelaide, 5000, South Australia, e-mail: jackbding@icloud.com

Submitted: 25.09.2021; Accepted: 07.10.2021 
flammatory changes are at least partly responsible for OA pain [10].

These findings are, however, complicated by inconsistencies between radiographic OA changes and actual symptoms, with one study suggesting the prevalence of radiographically diagnosed knee OA to be $28 \%$, but the overall prevalence of symptomatic knee OA to be $17 \%$ [11]. Therefore, some commentators have suggested that the mechanisms of OA are ultimately due to an interplay between various peripheral and central mechanisms [2].

While there are several potentially disease-modifying osteoarthritis drugs in phase 2/3 development, the current non-operative treatment objective revolves around pain reduction and therefore functional improvement, with limited emphasis on modifying disease course [12].

First-line pharmacologic options for osteoarthritic knee pain include oral analgesics and anti-inflammatory drugs. While acetaminophen is frequently used as a first-line option, its efficacy is limited, with a 2019 meta-analysis concluding that it generated minimal improvement in pain in knee OA [13].

Nonsteroidal anti-inflammatory drugs are also a first-line option, though they pose a risk of nephrotoxicity or gastrointestinal complications such as bleeding, thus limiting their utility in some patients [14]. While cyclooxygenase-2 selective inhibitors are an option for reducing gastrointestinal bleeding risk, they may impose greater financial burden on the patient and are associated with increased cardiovascular complications [15].

There are various intra-articular injectable therapies available for osteoarthritic knee pain, including corticosteroids (CSs), hyaluronic acid (HA), and platelet-rich plasma (PRP). Corticosteroids modulate nuclear steroid receptors, which leads to interruption of the inflammatory and immune cascade at multiple levels, and ultimately anti-inflammatory effects [16]. Specifically, CSs:

- impair inflammatory cell adhesion and migration through vascular endothelium,

- interrupt cell-to-cell communication by altering cytokine release, namely interleukin 1 (IL-1),

- impair leukotriene and prostaglandin synthesis,

- inhibit production of neutrophil superoxide and matrix metalloproteinase,

- reduce immunoglobulin synthesis [16].

Hyaluronic acid is secreted by various cells into the knee joint as a component of synovial fluid and the cartilage matrix, where it functions as a lubricant and shock absorber and regulates intracellular activities such as cytokine release [17].

Joints affected by OA typically have lower concentrations of HA in the synovial fluid compared to non-osteo- arthritic joints because synovial inflammation increases HA permeability [18, 19]. Viscosupplementation is the procedure by which HA is injected into joints. Supplementation of intra-articular HA is thought to restore normal levels of shock absorption and joint lubrication and to decrease inflammation, leading to chondroprotective effects [19].

Higher-molecular-weight $\mathrm{HA}$ has been associated with greater anti-inflammatory and proteoglycan synthesis effects compared to lower-molecular-weight options [20, 21]. One effect OA has on endogenous HA is degradation of originally high-molecular-weight $\mathrm{HA}$ (6500-10,900 kDa) into a lower weight (2700-4500 kDa), which could be one method by which pain is generated [22, 23].

One novel finding in animal models is that HA appears to directly inhibit nociceptors, which could be another mechanism for its analgesic effects [24]. Therapeutic use of PRP has been explored recently as a potential new treatment method for knee OA symptoms.

Platelets contain cytokines, chemokines, growth factors, and other proteins and molecules. They play a substantial and well-documented role in vasoconstriction, inflammation, angiogenesis, tissue regeneration, and immune responses $[25,26]$. In the context of OA, PRP has been suggested to affect bone and vessel remodelling, inflammation modulation, and collagen synthesis [27].

Oxygen ozone and stem cells injections have recently been proposed as possible alternatives to the abovementioned injectable options for the alleviation of pain and functional impairment in knee OA $[28,29]$.

The therapeutic effect of ozone therapy has been suggested to be due to local oxidative stress produced by ozone. While severe oxidative stress induces an inflammatory response with associated tissue injury through activation of nuclear transcription factor kappa B; moderate oxidative stress activates nuclear factor-erythroid 2-related factors, which induce an antioxidant response element, resulting in anti-inflammatory action [30]. Mesenchymal stem cells (MSC) are thought to give rise to all mature cartilage-, bone-, and fat tissue-producing cells and have been the target of a potential OA treatment aimed at reducing the loss of cartilage [29].

Operative options such as arthroplasty are generally reserved for patients with chronic and debilitating pain despite optimal pharmacologic therapy [15].

Table I below depicts the current recommendations for non-operative options for treating knee OA pain from the American College of Rheumatology (ACR) and Arthritis Foundation [31], and the American Academy of Orthopaedic Surgeons (AAOS) [32].

As seen in Table I, there is a notable discrepancy in recommendation between 2 associations with regards 
Table I. Selected academic recommendations from the American College of Rheumatology/Arthritis Foundation 2019 and American Academy of Orthopaedic Surgeons 2021 guidelines for knee osteoarthritis management

\begin{tabular}{|lcc|}
\hline \multicolumn{3}{|c|}{ Selected academic recommendations } \\
\hline Treatment option & ACR/Arthritis Foundation 2019 & AAOS 2021 \\
\hline Supervised exercise & Strongly recommended & Strong evidence \\
\hline Weight loss & Strongly recommended & Moderate evidence \\
\hline Acupuncture & Conditionally recommended & Limited evidence \\
\hline Knee brace & Strongly recommended & Moderate evidence \\
\hline Oral NSAIDs & Strongly recommended & Strong evidence \\
\hline Topical NSAIDs & Strongly recommended & Strong evidence \\
\hline Acetaminophen & Conditionally recommended & Strong evidence \\
\hline Intra-articular steroids & Strongly recommended & Moderate evidence \\
\hline Hyaluronic acid & Conditionally recommended against & Moderate evidence \\
\hline Platelet-rich plasma & Strongly recommended against & Limited evidence \\
\hline Oxygen ozone injections & No evaluation & No evaluation \\
\hline Stem cell injections & Strongly recommend against & No evaluation \\
\hline
\end{tabular}

AAOS - American Academy of Orthopaedic Surgeons, ACR - American College of Rheumatology, NSAIDs - non-steroidal anti-inflammatory drugs.

to intra-articular steroid, HA, or PRP administration. The aim of this paper is to review the available literature and to provide a broad overview of the efficacy and safety profiles of the injectable therapies available for alleviating osteoarthritic knee pain.

\section{Material and methods}

A search for papers published in PubMed was conducted in May 2021. The following search terms were used: "knee osteoarthritis" and "hyaluronic acid" or "platelet-rich plasma" or "steroids" or "oxygen ozone" or "stem cell", and combinations thereof. The abstracts and reference section of primary and secondary literature papers were reviewed manually.

Studies that were randomized controlled trials or meta-analyses were selected for further review. Papers that were irrelevant, duplicate, or not published in the English language were excluded from further review.

Table II below shows an overview of the primary literature reviewed in this study.

Table III below shows an overview of the secondary literature reviewed in this study.

\section{Intra-articular corticosteroids}

Intra-articular corticosteroid injections are used internationally, although guidelines vary with regards to their recommendations about its efficacy. The 2019 ACR/ Arthritis Foundation strongly recommend their use in knee OA, whereas the AAOS state there is only moderate evidence to support its use [31, 32].
A 2020 meta-analysis of 8 randomized control trials (RCTs) concluded that intra-articular CS have a significantly positive effect on knee pain in the short-term after injection, with its positive effects becoming non-significant after the 4-month mark [33]. While earlier studies have noted short-term improvement in pain following CS injections, some suggested the improvement could be significantly sustained up to 24 weeks post-injection [34, 35].

A 2015 meta-analysis of 27 RCTs concluded that, depending on the timeframe, CS may be superior to placebo administration for pain reduction. After stratifying the data set according to the time elapsed since the initial intervention, the greatest benefits appeared within the first 1 to 2 weeks, with an SMD of -0.48 (95\% $\mathrm{Cl}:-0.7$ to -0.27$)$. The benefits decreased at the 4-to6-week mark, then decreased further at 13 weeks, and finally became insignificant after 26 weeks; SMD -0.07 (95\% Cl: -0.25 to 0.11 ).

However, the RCTs included in the analysis were generally of high or uncertain risk of bias, with small sample sizes and low methodological quality. Some of the included trials were quasi-randomized, and a true control group, such as intra-articular saline injection, was not a pre-requisite for inclusion [34]. The conclusion that CS injection transiently improves OA knee pain in the short term (4 weeks) is, however, corroborated by a recent, high-quality RCT [36].

One recent $\mathrm{RCT}$ of moderate size with an adequate randomization process, 2-way blinding, and an intention-to-treat analysis concluded that there was no significant pain benefit in CS injection prior to commencing a graded-exercise program. The main limitation of 


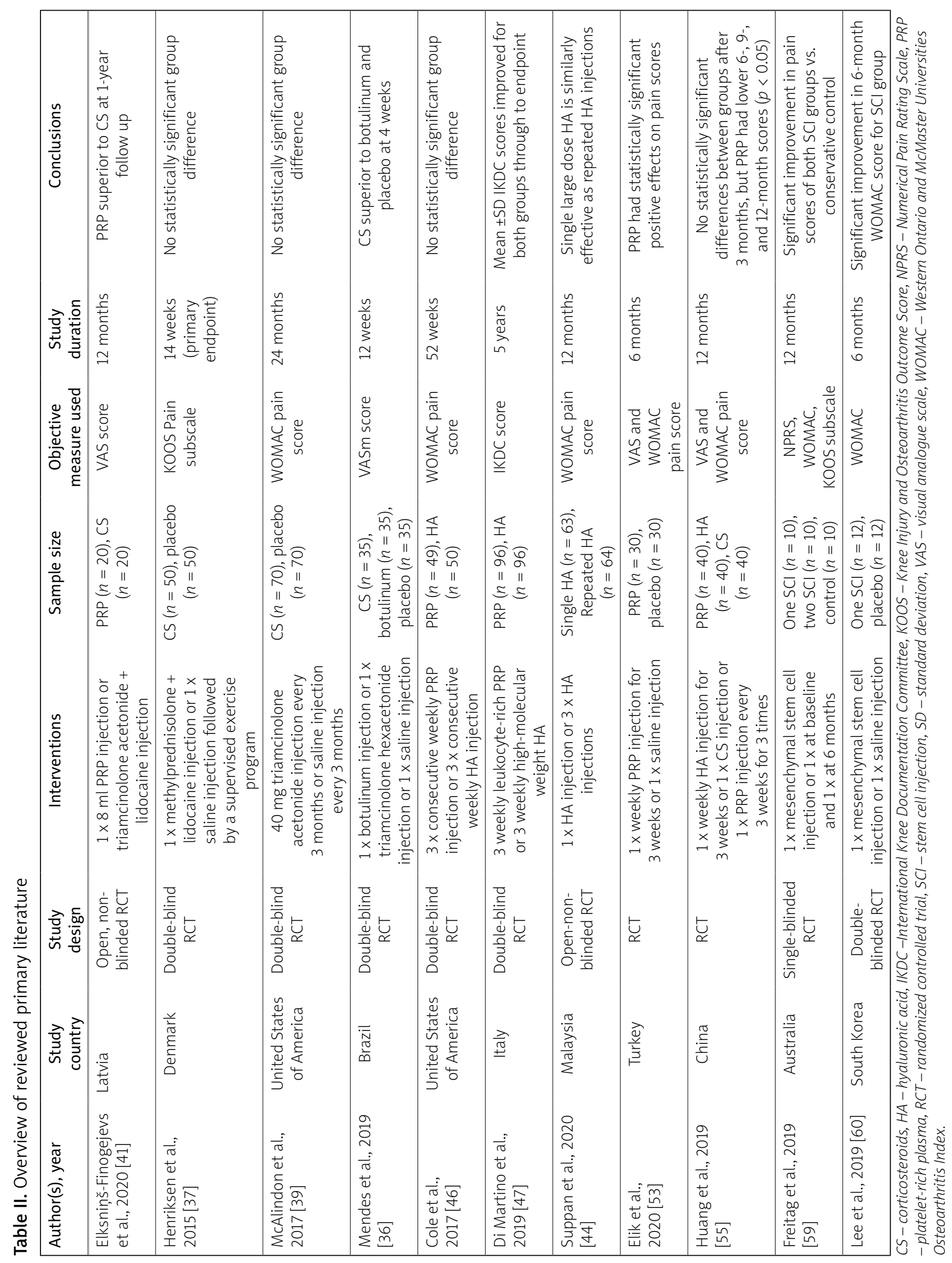




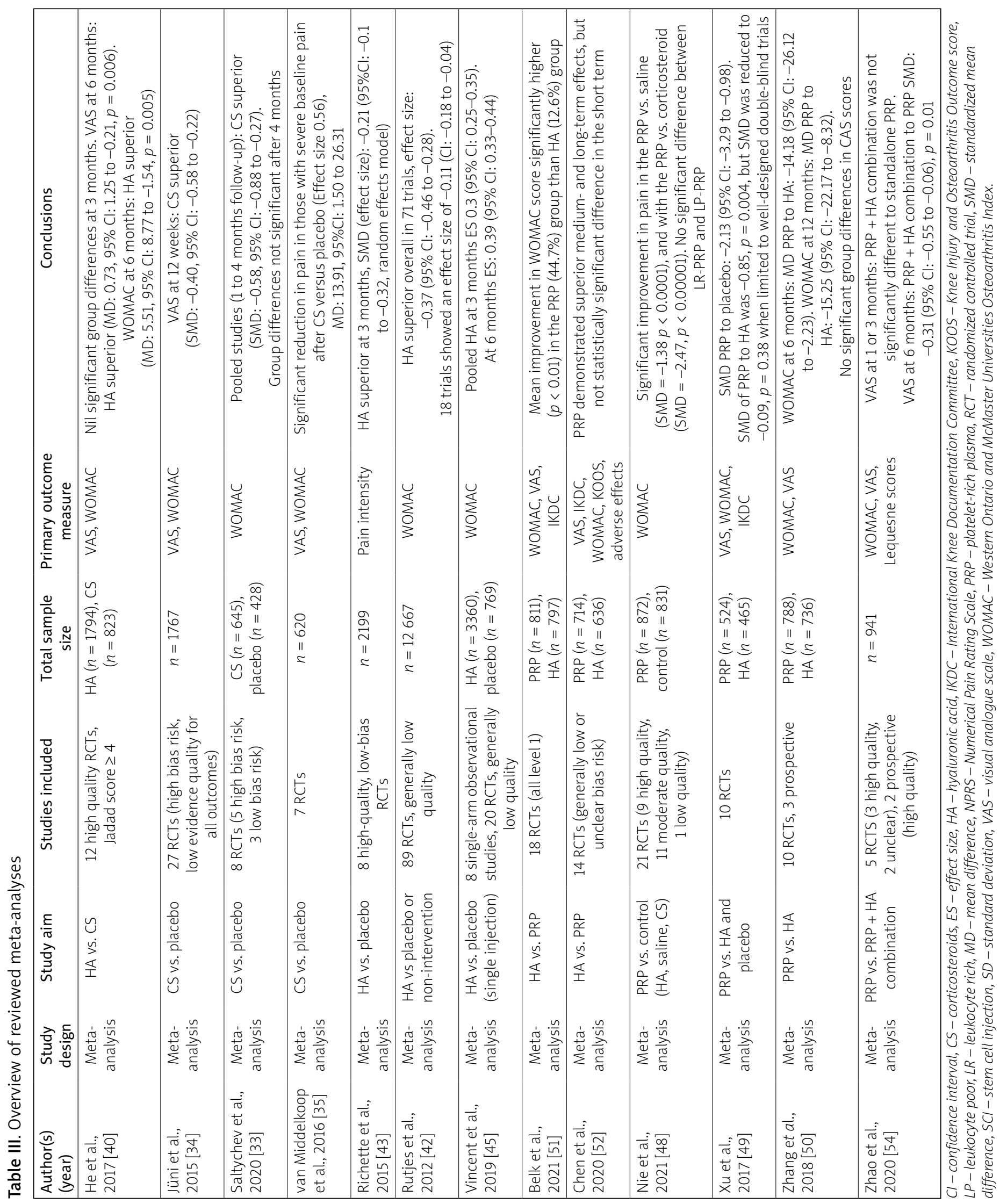


this study was that the single CS dose chosen was on the lower end of recommended dosages, and therefore a dose-response relationship is unknown [37].

This trial brings about significant real-world implications of CS injections. It differed in that most comparable trials focused on participant pain experiences without a standardized exercise component. Notably, this is in the context of both ACR/Arthritis Foundation and AAOS strongly recommending or citing strong evidence for a supervised exercise program in treating osteoarthritic pain [31]. Exploring the effect of CS on OA knee pain in the context of exercise may be an area for further exploration.

There is an ongoing paucity of research that explores the long-term efficacy and safety of repeated corticosteroid injections. One prospective, non-experimental cohort study examined 318 participants with radiographic knee OA, who initiated either CS injections over a 2-year period. They concluded that CS injections were not associated with continuous symptom reduction compared to non-users over a 2-year period [38].

This conclusion corroborates that of an earlier double-blind RCT by McAlindon et al. [39], including 140 participants who were subjected to either CS or saline injections every 3 months for a period of 2 years. Notably, McAlindon also quantitatively evaluated cartilage volume, and discovered that triamcinolone resulted in significantly greater volume loss of articular cartilage. This raises the possibility that repeated CS administrations over time may be detrimental in terms of OA disease progression without significant improvement in pain, which may be an area for future study.

Comparison studies between intra-articular CS injections and other intra-articular treatment modalities have been performed. A recent meta-analysis of 12 RCTs comparing CS with HA concluded that CS resulted in greater improvement in VAS score up to 1 month, but HA was more effective at the 6-month follow-up, with an equal gain of function [40].

A 2020 RCT comparing CS and PRP found that a single PRP injection demonstrated a statistically significant improvement in pain in patients with moderate knee arthritis as measured by VAS, IKDC, and KSS scales compared to CS at 1 year. While both the CS and PRP groups reported improved pain scores up to 5 weeks without significant group differences, the improvement was only sustained in the PRP group. This study was primarily limited by the lack of blinding of both participants and clinicians [41].

\section{Hyaluronic acid}

There are several meta-analyses that evaluate the efficacy of viscosupplementation for osteoarthritic knee pain. A 2012 meta-analysis of 89 RCTs evaluating HA injections vs. placebo concluded that while 71 trials showed a moderate reduction in pain, with an effect size for pain -0.37 (95\% Cl: -0.46 to -0.28$)$, 18 large trials within the same meta-analysis had an effect size for pain of -0.11 ( $\mathrm{Cl}$ : -0.18 to -0.04$)$, which was regarded as not clinically relevant [42].

However, the trials included in this study were generally of very low quality. A later 2015 meta-analysis of 8 high-quality RCTs yielded lower pain estimates at 3 months, with an effect size of $0.21(95 \% \mathrm{Cl}$ : -0.1 to -0.32) [43]. This discrepancy could be due to the inclusion of lower-quality trials that lacked adequate blinding and randomization processes in the earlier meta-analysis.

A more recent RCT by Suppan et al. [44] compared single dose vs. repeated doses of HA injections and found that there was no significant difference between groups for pain scores up to 12 months. These results were corroborated by a recent 2019 meta-analysis of 28 studies, which concluded that single HA injections produced results comparable to those of multiple injections [45]. Because there is an approximately 30\% lower cost associated with single-dose vs. multiple-dose regimes of $\mathrm{HA}$, a single larger dose of HA is suggested as a future point of exploration [44].

The efficacy of HA injections has also been compared with that of PRP. Cole et al. [46], conducted a double-blind RCT with a total sample size of 99 , and discovered no difference in WOMAC domains between HA and PRP groups at any time point up to 52 weeks, although significant improvements were observed in IKDC scores in the PRP group compared to HA.

In the same study, Cole et al. [46], also conducted a biochemical analysis and discovered that the differences between the PRP and HA groups for 2 cytokines, IL-1 $\beta$ and tumour necrosis factor $\alpha$, differed, with the PRP group having significantly lower cytokine levels. One possible explanation for these biologic changes is perhaps the anti-inflammatory properties of PRP, which could be an area for further exploration.

Di Martino et al. [47], conducted a 5-year double blinded RCT analysing the long-term efficacy of repeated HA compared to repeated PRP interventions. They noted significant improvement in pain and function at endpoint in both groups, although the percentage of participants requiring reintervention at 24 months was significantly higher in the HA group (37.1\% vs. $22.6 \%, p=0.036$ ).

These results differ to those of Liu et al. [38], who conducted a longitudinal study of 412 participants, about $20 \%$ of whom used hyaluronic injections and around $78 \%$ used CS injections, and concluded that neither group resulted in sustained symptom relief at 2-year follow-up compared to non-users. 
However, Liu's study was severely limited by a lack of standardization with dosages and formulations of interventions. In addition, there may be discrepancy between time of intervention and pain assessment between participants. These results suggest that while low-quality evidence supports the benefit of HA, greater clinical effect may be seen with PRP.

\section{Platelet-rich plasma}

The meta-analyses that examine the efficacy of PRP injections are heterogenous in their conclusions [48-50]. A 2021 meta-analysis of 21 RCTs that examined PRP vs. HA/CS/saline control groups concluded that the PRP group had significant pain improvements over the CS (SMD $=-2.47)$ and saline $(S M D=-1.38)$ groups, but the high heterogeneity in data between the PRP and HA groups led to inconclusive interpretations [48].

This conclusion contradicts that of a 2017 meta-analysis [49], which stated that while their PRP group did have significantly superior pain scores to the HA group in their overall analysis, if only high-quality double-blinded RCTs were analysed, the differences became insignificant.

They inferred that the supposed pain benefits of PRP over HA in most individual trials was probably secondary to inadequate blinding techniques. Regardless, they still concluded PRP to be superior to placebo, with the differences between groups being -2.13 (95\% Cl: -3.29 to -0.98). A 2018 meta-analysis discovered that PRP significantly reduced WOMAC pain scores compared to HA, but the significance was lost with VAS pain scoring [50].

A common denominator with all abovementioned meta-analyses is the scarcity of high-quality RCTs with double-blinding methods.

Xu et al. [49] included only double-blinded RCTs in their calculations, which resulted in only 2 RCTs out of an overall 10 RCTs remaining eligible for analysis, and therefore their conclusion of statistical non-significance between PRP and HA pain reduction rested on a very limited sample size.

Belk et al. [51] performed a meta-analysis of 18 level-1 studies comparing PRP with HA. Mean improvement in total WOMAC score was significantly higher in the PRP (44.7\%) compared to the HA group (12.6\%).

Chen et al. [52] had similar findings in their meta-analysis, noting that PRP demonstrated superior pain score reductions in the medium (12 to 24 weeks) and long term (> 24 weeks), but short-term differences were not statistically significant. Again, both these meta-analyses [51, 52] are limited by RCTs of generally low or unclear bias risk.

A recent 2020 RCT with a total sample size of $60 \mathrm{com}$ pared the efficacy of PRP to placebo and found that pain scores of the PRP group significantly improved in the 1st and 6th month after intervention, while cartilage thickness was not significantly altered [53].

Further studies have been performed exploring combination injectable formulations. Zhao et al. [54] performed a meta-analysis of studies comparing efficacy of PRP combined with HA vs. PRP or HA alone. They found that compared with PRP alone, PRP combined with HA resulted in improved WOMAC total scores and VAS scores at 6 months without any significant difference in adverse effects.

A common denominator between existing PRP studies is the diversity in preparation methods, dosages, and frequency of intervention, with a standard yet to be set. One RCT compared the effects of PRP, HA, and CS injection on VAS and WOMAC pain scores with follow-ups lasting up until 12 months. They found no statistically significant group differences at 3 months, but PRP had lower WOMAC scores at the 6-, 9-, and 12-month follow-ups $(p<0.05)[55]$

While the major intra-articular injectables were compared within the same trial, the method used to prepare PRP may vary between trials, which may have critical implications for interpretation. Indeed, some studies have identified leukocyte-poor PRP being associated with greater efficacy than leukocyte-rich preparations [51].

The reasoning for this has been suggested as being due to elevated leukocyte concentrations being associated with elevated catabolic cytokines, which potentially counteract the effect of the anabolic cytokines contained within platelets [56].

At present, there is a paucity of literature that directly compares the efficacy of different PRP preparations based on leukocyte concentration, which is a possible point for future studies.

\section{Other injectable therapies}

Oxygen ozone and stem cell injections have been proposed as being viable alternatives for improving pain and function in knee OA. Current data tentatively suggest that oxygen ozone therapy may improve osteoarthritic knee pain and function in the short-term [57].

However, available RCTs on oxygen ozone therapy for knee OA are limited by poor methodological quality and are flawed by relevant bias. Stem cell injections have also been introduced as an emerging therapy. A 2017 systematic review identified 6 trials suggesting superior radiological and histological outcomes with no adverse effects; however, these trials all had high risk of bias and low-quality evidence [58].

More recently, a 2019 RCT performed by Freitag et al. [59] demonstrated clinically significant pain and functional improvement at 12 months with adipose MSC, 
with modification of disease progression with radiological analysis using the Magnetic Resonance Imaging Osteoarthritis Knee Score, with no significant adverse effects. However, the sample size was small, with a total of 30 participants dispersed between 3 groups. Notably, the biologic alterations pose the possibility of disease modifying processes and may be worthwhile for further exploration.

Lee et al. [60] demonstrated a significant improvement in WOMAC score with a single injection of adipose MSC; however, the sample size was small $(n=12)$. Overall, there is a scarcity of high-quality studies evaluating oxygen ozone and stem cell injections in relation to OA knee pain. Further, higher-quality RCTs with adequate blinding, larger sample sizes, and longer-term follow-up are needed to acquire more substantiated knowledge on their long-term safety and efficacy.

\section{Conclusions}

Intra-articular corticosteroid, hyaluronic, or PRP injections can provide short-term to medium-term (4 to 12 weeks) improvement in pain and function as measured by either WOMAC and/or VAS scores with minimal incidence of serious adverse events. Out of the above, the evidence behind CS for pain relief is most substantiated. There is a scarcity of head-to-head comparisons between each injectable therapy, although there is some emerging evidence that suggest possibly superior pain reduction with HA over the long-term (6 months to 1 year) compared to CS.

Some trials also tentatively demonstrated superior and sustained pain improvements with PRP over the longer term (6 months to 1 year), although they are limited by their small sample sizes and quality.

In addition, current evaluation of PRP is severely limited by the heterogeneity in its preparation and injection techniques between trials, which makes it difficult to make a blanket statement regarding its efficacy.

Ozone therapy and mesenchymal stem cell injections have an emerging evidence base behind them. Larger high-quality trials comparing their effects on knee pain and function with other injectable therapies is needed before any significant conclusion can be made regarding their safety or efficacy.

The authors declare no conflict of interest.

\section{References}

1. Lawrence RC, Felson DT, Helmick CG, et al. Estimates of the prevalence of arthritis and other rheumatic conditions in the United States. Part II. Arthritis Rheum 2008, 58: 26-35; DOI: 10.1002/art.23176
2. Neogi T. The epidemiology and impact of pain in osteoarthritis. Osteoarthritis Cartilage 2013; 21: 1145-1153, DOI: 10.1016/j. joca.2013.03.018.

3. Guccione AA, Felson DT, Anderson JJ, et al. The effects of specific medical conditions on the functional limitations of elders in the Framingham Study. Am J Public Health 1994; 84: 351358, DOI: 10.2105/ajph.84.3.351.

4. Kawano MM, Araújo IL, Castro MC, Matos MA. Assessment of quality of life in patients with knee osteoarthritis. Acta Ortop Bras 2015; 23: 307-310, DOI: 10.1590/1413785220152306150596.

5. van Dijk GM, Veenhof C, Lankhorst GJ, Dekker J. Limitations in activities in patients with osteoarthritis of the hip or knee: the relationship with body functions, comorbidity and cognitive functioning. Disabil Rehabil 2009; 31: 1685-1691, DOI: 10.1080/09638280902736809.

6. Lementowski PW, Zelicof SB. Obesity and osteoarthritis. Am J Orthop (Belle Mead NJ) 2008; 37: 148-151.

7. Raja SN, Carr DB, Cohen M, et al. The revised International Association for the Study of Pain definition of pain: concepts, challenges, and compromises. Pain 2020; 161: 1976-1982, DOI: 10.1097/j.pain.0000000000001939.

8. Mobasheri A, Batt M. An update on the pathophysiology of osteoarthritis. Ann Phys Rehabil Med 2016; 59: 333-339, DOI: 10.1016/j.rehab.2016.07.004.

9. Miller RE, Miller RJ, Malfait AM. Osteoarthritis joint pain: the cytokine connection. Cytokine 2014; 70: 185-193, DOI: 10.1016/j.cyto.2014.06.019.

10. Nees TA, Rosshirt N, Zhang JA, et al. Synovial cytokines significantly correlate with osteoarthritis-related knee pain and disability: inflammatory mediators of potential clinical relevance. J Clin Med 2019; 8: 1343, DOI: 10.3390/jcm8091343.

11. Jordan JM, Helmick CG, Renner JB, et al. Prevalence of knee symptoms and radiographic and symptomatic knee osteoarthritis in African Americans and Caucasians: the Johnston County Osteoarthritis Project. J Rheumatol 2007; 34: 172-180.

12. Oo WM, Hunter DJ. Disease modification in osteoarthritis: are we there yet? Clin Exp Rheumatol 2019; 37: 135-140.

13. Leopoldino AO, Machado GC, Ferreira PH, et al. Paracetamol versus placebo for knee and hip osteoarthritis. Cochrane Database Syst Rev 2019; 2: CD013273, DOI: 10.1002/14651858. Cd013273.

14. Lazzaroni M, Bianchi Porro G. Gastrointestinal side-effects of traditional non-steroidal anti-inflammatory drugs and new formulations. Aliment Pharmacol Ther 2004; 20: 48-58, DOI: 10.1111/j.1365-2036.2004.02037.x.

15. Sinusas K. Osteoarthritis: diagnosis and treatment. Am Fam Physician 2012; 85: 49-56.

16. Uthman I, Raynauld JP, Haraoui B. Intra-articular therapy in osteoarthritis. Postgrad Med J 2003; 79: 449-453, DOI: 10.1136/ pmj.79.934.449.

17. Iannitti T, Lodi D, Palmieri B. Intra-articular injections for the treatment of osteoarthritis: focus on the clinical use of hyaluronic acid. Drugs R D 2011; 11: 13-27, DOI: 10.2165/11539760000000000-00000.

18. Migliore A, Procopio S. Effectiveness and utility of hyaluronic acid in osteoarthritis. Clin Cases Miner Bone Metab 2015; 12: 31-33, DOI: 10.11138/ccmbm/2015.12.1.031. 
19. Altman RD, Manjoo A, Fierlinger A, et al. The mechanism of action for hyaluronic acid treatment in the osteoarthritic knee: a systematic review. BMC Musculoskelet Disord 2015; 16 : 321, DOI: 10.1186/s12891-015-0775-z.

20. Elmorsy S, Funakoshi T, Sasazawa F, et al. Chondroprotective effects of high-molecular-weight cross-linked hyaluronic acid in a rabbit knee osteoarthritis model. Osteoarthritis Cartilage 2014; 22: 121-127, DOI: 10.1016/j.joca.2013.10.005.

21. Lajeunesse D, Delalandre A, Martel-Pelletier J, Pelletier JP. Hyaluronic acid reverses the abnormal synthetic activity of human osteoarthritic subchondral bone osteoblasts. Bone 2003; 33: 703-710, DOI: 10.1016/s8756-3282(03)00206-0.

22. Bowman S, Awad ME, Hamrick MW, et al. Recent advances in hyaluronic acid based therapy for osteoarthritis. Clin Transl Med 2018; 7: 6, DOI: 10.1186/s40169-017-0180-3.

23. Band PA, Heeter J, Wisniewski HG, et al. Hyaluronan molecular weight distribution is associated with the risk of knee osteoarthritis progression. Osteoarthritis Cartilage 2015; 23 70-76, DOI: 10.1016/j.joca.2014.09.017.

24. Gomis A, Miralles A, Schmidt RF, Belmonte C. Intra-articular injections of hyaluronan solutions of different elastoviscosity reduce nociceptive nerve activity in a model of osteoarthritic knee joint of the guinea pig. Osteoarthritis Cartilage 2009; 17 798-804, DOI: 10.1016/j.joca.2008.11.013.

25. Gato-Calvo L, Magalhaes J, Ruiz-Romero C, et al. Platelet-rich plasma in osteoarthritis treatment: review of current evidence. Ther Adv Chronic Dis 2019; 10: DOI: 10.1177/2040622319825567.

26. Alves R, Grimalt R. A review of platelet-rich plasma: history, biology, mechanism of action, and classification. Skin Appendage Disord 2018; 4: 18-24, DOI: 10.1159/000477353.

27. Sundman EA, Cole BJ, Karas V, et al. The anti-inflammatory and matrix restorative mechanisms of platelet-rich plasma in osteoarthritis. Am J Sports Med 2014; 42: 35-41, DOI 10.1177/0363546513507766.

28. de Sire A, Agostini F, Lippi L, et al. Oxygen-ozone therapy in the rehabilitation field: state of the art on mechanisms of action, safety and effectiveness in patients with musculoskeletal disorders. Biomolecules 2021; 11: 356, DOI: 10.3390/ biom 11030356

29. Whittle SL, Johnston RV, McDonald S, et al. Stem cell injections for osteoarthritis of the knee. Cochrane Database Syst Rev 2019; 2019: CD013342, DOI: 10.1002/14651858.CD013342.

30. Sagai M, Bocci V. Mechanisms of action involved in ozone therapy: is healing induced via a mild oxidative stress? Med Gas Res 2011; 1: 29, DOI: 10.1186/2045-9912-1-29.

31. Kolasinski SL, Neogi T, Hochberg MC, et al. 2019 American College of Rheumatology/Arthritis Foundation Guideline for the management of osteoarthritis of the hand, hip, and knee. Arthritis Care Res (Hoboken) 2020; 72: 149-162, DOI: 10.1002/ acr.24131.

32. American Academy of Orthopaedic Surgeons: management of osteoarthritis of the knee (non-arthroplasty) evidence-based clinical practice guideline, $3^{\text {rd }}$ ed. Available from: https://www. aaos.org/oak3cpg (Accessed: 18.08.2021)

33. Saltychev M, Mattie R, McCormick Z, Laimi K. The magnitude and duration of the effect of intra-articular corticosteroid injections on pain severity in knee osteoarthritis: a systematic review and meta-analysis. Am J Phys Med Rehabil 2020; 99: 617-625, DOI: 10.1097/phm.0000000000001384.

34. Jüni P, Hari R, Rutjes AW, et al. Intra-articular corticosteroid for knee osteoarthritis. Cochrane Database Syst Rev 2015; 2015: CD005328, DOI: 10.1002/14651858.CD005328.pub3.

35. Van Middelkoop M, Arden NK, Atchia I, et al. The OA Trial Bank: meta-analysis of individual patient data from knee and hip osteoarthritis trials show that patients with severe pain exhibit greater benefit from intra-articular glucocorticoids. Osteoarthritis Cartilage 2016; 24: 1143-1152, DOI: 10.1016/j. joca.2016.01.983.

36. Mendes JG, Natour J, Nunes-Tamashiro JC, et al. Comparison between intra-articular Botulinum toxin type $A$, corticosteroid, and saline in knee osteoarthritis: a randomized controlled trial. Clin Rehabil 2019; 33: 1015-1026, DOI: 10.1177/0269215519827996.

37. Henriksen M, Christensen R, Klokker L, et al. Evaluation of the benefit of corticosteroid injection before exercise therapy in patients with osteoarthritis of the knee: a randomized clinical trial. JAMA Intern Med 2015; 175: 923-930, DOI: 10.1001/jamainternmed.2015.0461.

38. Liu SH, Dubé CE, Eaton CB, et al. Longterm effectiveness of intraarticular injections on patient-reported symptoms in knee osteoarthritis. J Rheumatol 2018; 45: 1316-1324, DOI: 10.3899/jrheum.171385.

39. McAlindon TE, LaValley MP, Harvey WF, et al. Effect of intra-articular triamcinolone vs. saline on knee cartilage volume and pain in patients with knee osteoarthritis: a randomized clinical trial. JAMA 2017; 317: 1967-1975, DOI: 10.1001/ jama.2017.5283.

40. He WW, Kuang MJ, Zhao J, et al. Efficacy and safety of intraarticular hyaluronic acid and corticosteroid for knee osteoarthritis: a meta-analysis. Int J Surg 2017; 39: 95-103, DOI: 10.1016/j.ijsu.2017.01.087.

41. Elksniņš-Finogejevs A, Vidal L, Peredistijs A. Intra-articular platelet-rich plasma versus corticosteroids in the treatment of moderate knee osteoarthritis: a single-center prospective randomized controlled study with a 1-year follow up. J Orthop Surg Res 2020; 15: 257, DOI: 10.1186/s13018-020-01753-z.

42. Rutjes AW, Jüni P, da Costa BR, et al. Viscosupplementation for osteoarthritis of the knee: a systematic review and metaanalysis. Ann Intern Med 2012; 157: 180-191, DOI: 10.7326/ 0003-4819-157-3-201208070-00473.

43. Richette P, Chevalier X, Ea HK, et al. Hyaluronan for knee osteoarthritis: an updated meta-analysis of trials with low risk of bias. RMD Open 2015; 1: e000071, DOI: 10.1136/rmdopen-2015-000071.

44. Suppan VKL, Tew MM, Wong BC, et al. One-year follow-up of efficacy and cost of repeated doses versus single larger dose of intra-articular hyaluronic acid for knee osteoarthritis. J Orthop Surg (Hong Kong) 2020; 28: DOI: 10.1177/2309499019895029.

45. Vincent P. Intra-articular hyaluronic acid in the symptomatic treatment of knee osteoarthritis: a meta-analysis of single-injection products. Curr Ther Res Clin Exp 2019; 90: 39-51, DOI: 10.1016/j.curtheres.2019.02.003.

46. Cole BJ, Karas V, Hussey $\mathrm{K}$, et al. Hyaluronic acid versus platelet-rich plasma: a prospective, double-blind randomized controlled trial comparing clinical outcomes and ef- 
fects on intra-articular biology for the treatment of knee osteoarthritis. Am J Sports Med 2017; 45: 339-346, DOI: 10.1177/0363546516665809.

47. Di Martino A, Di Matteo B, Papio T, et al. Platelet-rich plasma versus hyaluronic acid injections for the treatment of knee osteoarthritis: results at 5 years of a double-blind, randomized controlled trial. Am J Sports Med 2019; 47: 347-354, DOI: 10.1177/0363546518814532.

48. Nie LY, Zhao K, Ruan J, Xue J. Effectiveness of platelet-rich plas$\mathrm{ma}$ in the treatment of knee osteoarthritis: a meta-analysis of randomized controlled clinical trials. Orthop J Sports Med 2021; 9: 2325967120973284, DOI: 10.1177/2325967120973284.

49. Xu Z, Luo J, Huang X, et al. Efficacy of platelet-rich plasma in pain and self-report function in knee osteoarthritis: a best-evidence synthesis. Am J Phys Med Rehabil 2017; 96: 793-800, DOI: $10.1097 / \mathrm{phm} .0000000000000746$.

50. Zhang HF, Wang CG, Li H, et al. Intra-articular platelet-rich plasma versus hyaluronic acid in the treatment of knee osteoarthritis: a meta-analysis. Drug Des Devel Ther 2018; 12 445-453, DOI: 10.2147/dddt.S156724.

51. Belk JW, Kraeutler MJ, Houck DA, et al. Platelet-rich plasma versus hyaluronic acid for knee osteoarthritis: a systematic review and meta-analysis of randomized controlled trials. Am J Sports Med 2021; 49: 249-260, DOI: 10.1177/0363546520909397.

52. Chen Z, Wang C, You D, et al. Platelet-rich plasma versus hyaluronic acid in the treatment of knee osteoarthritis: a meta-analysis. Medicine (Baltimore) 2020; 99: e19388, DOI: 10.1097/md.0000000000019388.

53. Elik H, Doğu B, Yılmaz F, et al. The efficiency of platelet-rich plasma treatment in patients with knee osteoarthritis. J Back Musculoskelet Rehabil 2020; 33: 127-138, DOI: 10.3233/bmr181374
54. Zhao J, Huang H, Liang G, et al. Effects and safety of the combination of platelet-rich plasma (PRP) and hyaluronic acid $(\mathrm{HA})$ in the treatment of knee osteoarthritis: a systematic review and meta-analysis. BMC Musculoskelet Disord 2020; 21: 224, DOI: 10.1186/s12891-020-03262-w.

55. Huang Y, Liu X, Xu X, Liu J. Intra-articular injections of platelet-rich plasma, hyaluronic acid or corticosteroids for knee osteoarthritis: a prospective randomized controlled study. Orthopade 2019; 48: 239-247, DOI: 10.1007/s00132-01803659-5.

56. Sundman EA, Cole BJ, Fortier LA. Growth factor and catabolic cytokine concentrations are influenced by the cellular composition of platelet-rich plasma. Am J Sports Med 2011; 39: 2135-2140, DOI: 10.1177/0363546511417792.

57. Sconza C, Respizzi S, Virelli L, et al. Oxygen-ozone therapy for the treatment of knee osteoarthritis: a systematic review of randomized controlled trials. Arthroscopy 2020; 36: 277-286, DOI: 10.1016/j.arthro.2019.05.043.

58. Pas HI, Winters M, Haisma HJ, et al. Stem cell injections in knee osteoarthritis: a systematic review of the literature. Br J Sports Med 2017; 51: 1125-1133, DOI: 10.1136/ bjsports-2016-096793.

59. Freitag J, Bates D, Wickham J, et al. Adipose-derived mesenchymal stem cell therapy in the treatment of knee osteoarthritis: a randomized controlled trial. Regen Med 2019; 14 : 213-230, DOI: 10.2217/rme-2018-0161.

60. Lee WS, Kim HJ, Kim KI, et al. Intra-articular injection of autologous adipose tissue-derived mesenchymal stem cells for the treatment of knee osteoarthritis: a phase $\mathrm{llb}$, randomized, placebo-controlled clinical trial. Stem Cells Transl Med 2019; 8: 504-511, DOI: 10.1002/sctm.18-0122. 